\title{
Transatlantica
}

Revue d'études américaines. American Studies Journal

\section{Le militantisme politique aux Etats-Unis au lendemain du 11 septembre 2001}

\section{Marianne Debouzy}

\section{(2) OpenEdition}

1 Journals

\section{Édition électronique}

URL : http://journals.openedition.org/transatlantica/2883

DOI : $10.4000 /$ transatlantica.2883

ISSN : 1765-2766

Éditeur

AFEA

\section{Référence électronique}

Marianne Debouzy, « Le militantisme politique aux Etats-Unis au lendemain du 11 septembre 2001 », Transatlantica [En ligne], 1 | 2008, mis en ligne le 14 mai 2008, consulté le 29 avril 2021. URL : http:// journals.openedition.org/transatlantica/2883; DOI : https://doi.org/10.4000/transatlantica.2883

Ce document a été généré automatiquement le 29 avril 2021

\section{(c) (i) (9)}

Transatlantica - Revue d'études américaines est mis à disposition selon les termes de la licence Creative Commons Attribution - Pas d'Utilisation Commerciale - Pas de Modification 4.0 International. 


\title{
Le militantisme politique aux Etats- Unis au lendemain du 11 septembre 2001
}

\author{
Marianne Debouzy
}

1 Le long silence des dissenters face au déferlement patriotique d'après le 11 septembre, face au poids de l'autocensure, à l'absence d'opposition visible à la guerre aussi, au triomphe de l'idéologie conservatrice qui semblait avoir réduit au silence toute contestation, a soulevé bien des interrogations. Qu'en était-il du militantisme politique aux Etats-Unis?

2 Avant de tenter d'analyser la situation au lendemain des attaques contre les Twin Towers, il semble nécessaire d'éclaircir quelques notions. Quand on évoque le militantisme politique, de quoi parle-t-on? Le dictionnaire Robert définit un militant comme "quelqu'un qui combat, qui lutte», ce qui semble un peu réducteur. Un militant est quelqu'un qui est convaincu de la justesse d'une cause ou d'une doctrine et qui s'emploie à la défendre, à la répandre et à l'appliquer. L'expression « militantisme politique » a un caractère problématique car toute activité militante est politique dans un sens large, que l'on parle de la grève des janitors, de mouvements étudiants tels que United Against Sweatshops agissant contre l'exploitation par des grandes marques des travailleurs du Tiers Monde, ou encore des revendications des secrétaires des universités. Peut-on tenter une typologie des militants politiques? Etre militant, est-ce la même chose en France et aux Etats-Unis, dans les années 1930 et aujourd'hui ? La culture politique est différente selon les lieux, mais aussi selon les époques; l'identité $\mathrm{du}$ militant change avec le temps, tout comme la conception du militantisme. En France, on associe traditionnellement les militants à des organisations politiques, des partis, qui, à travers une idéologie, valorisent le collectif au détriment de l'individuel, et le sens du sacrifice ; le militant type, c'est le militant ouvrier, modèle qui nourrit le mythe prolétarien. Parce que les partis politiques de gauche sont inexistants aux EtatsUnis à une échelle nationale, il semble impossible de faire coïncider l'image du militant 
français avec celle du militant américain, même si les points communs entre ces deux modèles du militantisme font l'objet de réflexions ${ }^{1}$.

3 Ce travail, nécessairement incomplet, se fonde sur les manifestations de la mouvance progressiste qui ont eu lieu immédiatement après le 11 septembre, sur les manifestations contre la guerre en Afghanistan et en Irak ainsi que sur les manifestations anti-Bush pendant la campagne présidentielle de 2004. L'enjeu a été de repérer les groupes présents, de s'interroger sur les formes de militantisme dont ils étaient porteurs ainsi que sur la notion même de militants : le fait d'assister à ces manifestations suffit-il à créer des militants? Cette question rejoint la question plus large des spécificités du militantisme "à l'américaine », et ce sont bien ses réussites, ses difficultés et ses limites dans le contexte de la guerre contre le terrorisme, celui de la campagne électorale mais également, plus globalement, le contexte politique américain, qui seront étudiés ici.

Repères chronologiques

4 Dès le week-end du 15 septembre, des manifestations, certes limitées, ont eu lieu à New York, et le 21 septembre, plus d'une centaine de campus manifestaient, exigeant de G. W. Bush de la prudence dans sa volonté de punir les responsables des attentats. Les manifestations les plus importantes se sont tenues à New York et Berkeley, dans le silence des médias. Au même moment, un appel était lancé par le Washington Peace Center pour une manifestation en faveur de la paix. Un quotidien britannique faisait état d'une coalition de religieux, de gens du business et du show-business qui s'était constituée pour dénoncer toute riposte militaire aux atrocités du 11 septembre; leur texte était signé, entre autres, par Martin Sheen, Harry Belafonte ou Rosa Parks². Selon les sondages de l'époque, $90 \%$ de la population soutenait une action militaire.

5 Ces premières manifestations expriment avant tout un refus de la violence et de la vengeance : les pancartes aperçues dans la manifestation du 25 septembre à New York affichaient : "Our grief is not a cry for war », «Break the cycle of violence », «War is not the answer ", "It's time for reflection, not revenge " ou encore « An eye for an eye leaves the whole world blind». Un appel pour une manifestation à Washington le 29 septembre est lancé, signé par Ramsey Clark, ancien ministre de la justice de Lyndon Johnson, l'historien Howard Zinn, auteur de A People's History of the United States, parmi bien d'autres personnalités. Dès les premières manifestations apparaît le signe ANSWER (Act Now to Stop War and End Racism) sur les tracts diffusés par les militants de l'International Action Center.

6 Avant même que la guerre en Afghanistan ne soit déclenchée, donc, des manifestations expriment déjà une opposition à l'éventualité d'un conflit armé. Puis, avec la menace de la guerre, elles prennent une nouvelle ampleur : le 7 octobre 2001 une manifestation a lieu à New York à l'initiative de Not in Our Name, et 20000 personnes défilent, dont trois Prix Nobel de la paix. Est aussi présent Jim Creedon, un secouriste qui, bien que blessé le 11 septembre, est retourné dans une des deux Tours pour porter secours; le mouvement auquel il appartient, September Families for Peaceful Tomorrows, se définit ainsi : « Those are people who have lost loved ones and said 'Not in Our Name' ".

7 En 2002, lorsque la menace d'une guerre en Irak se fit jour, le même phénomène pouvait être observé : le 26 octobre 2002 des manifestations ont eu lieu à San Francisco et Washington. Ce jour-là, dans la capitale, la police indique officieusement à l'équipe de télévision Democracy Now qu'il y avait 150000 à 200000 personnes, tandis que le New York Times annonce, lui, qu'il y avait moins de monde que n'en attendaient les 
organisateurs; plus tard, le journal avouera qu'il n'était pas sur les lieux ${ }^{4}$. La guerre se rapproche et les manifestations s'intensifient: en novembre 2002, par exemple, des manifestations à répétition se déroulent à Washington, devant le siège des Nations Unies, et l'on peut lire des pancartes avec le slogan « No Blood for Oil». Mais la presse reste silencieuse face à des manifestants qui ont du mal à se faire entendre. Qui sontils?

Composition des manifestations

8 Certains groupes sont bien connus et étaient déjà actifs pendant la guerre du Vietnam. En premier lieu, les groupes pacifistes, dont certains sont religieux (American Friends Service Committee, un groupe Quaker; The Fellowship of Reconciliation, une organisation œcuménique inter-religieuse fondée en 1917 ; Pax Christi, une organisation catholique traditionnelle; Jonah House, une organisation catholique radicale fondée par Philip Berrigan, anti-guerre et anti-nucléaire) et d'autres laïcs (The War Resisters' League, la plus grande organisation pacifiste laïque fondée en 1922; The Women's International League for Peace and Freedom, organisation internationale avec des groupes locaux; Peace Action, une organisation née dans les années 1990 de la fusion de Freeze Movement et SANE).

9 Puis viennent les Eglises, fortement impliquées dans le mouvement anti-guerre : toutes les églises protestantes regroupées dans le National Council of Churches (66 millions de fidèles) sont représentées à l'exception de la Southern Baptist Convention, la plus puissante des églises évangéliques à dominance blanche avec ses 16 millions de fidèles, seule favorable à la guerre. Le National Council of Churches a envoyé des représentants en Irak dès septembre 2002 et s'est engagé dans des actions de désobéissance civile, bien que tous les fidèles n'aient pas suivi. L'église catholique, dont les évêques rejettent la guerre, a une aile modérée et une aile radicale. Enfin, dans les manifestations, les représentants institutionnels des Juifs semblent s'être faits discrets, et les représentants des Musulmans encore davantage.

Les autres groupes, dont certains ont joué un rôle moteur dans l'organisation des manifestations, sont essentiellement des organisations politiques de gauche. The International Action Center, par exemple, présent dès les premières manifestations, a été fondé par Ramsey Clark, ministre de la justice de mars 1967 à janvier 1969 et personnage controversé : opposant aux guerres du Vietnam, du Golfe et d'Irak, il a successivement pris la défense de Saddam Hussein, Milosevic ou encore Mumia AbuJamal; il a mis en cause l'attitude des Etats-Unis sur les questions de la Palestine, de Cuba et de la Corée. The International Action Center est contrôlé par ANSWER dont le brain-trust inclut des membres de deux groupes d'extrême-gauche, l'un issu d'une scission à l'intérieur du Socialist Workers' Party, le Working World Party, trotskyste, et l'autre maoïste, The Revolutionary Communist Party. On dit aussi que figurent dans le groupe des dissidents du PC américain. Il s'agit certes de groupuscules, mais avec une certaine expérience du terrain et une évidente capacité organisationnelle. Présente également, l'organisation Not in Our Name, déjà mentionnée, formée à l'origine par des parents de victimes mais dont on dit qu'elle a été prise en main, fin 2002, par le Revolutionary Communist Party, à New York. Elle a rassemblé des milliers de signatures sur une pétition devenue culte qui appelle à résister à de nouvelles mesures liées à la politique globale menée depuis le 11 septembre. Début 2002, le texte comptait 4000 signatures ; à la fin de la même année, il en avait recueilli 30000. 
11 Une multitude d'autres organisations et associations participent aux manifestations: celles du mainstream, dont beaucoup s'adressent à tous, telles United for Peace, Win without War, Common Cause (soutenue par Arthur Schlesinger et Walter Cronkite), ou encore MoveOn, fondée en 1998 sur internet. D'autres représentent des groupes particuliers: les artistes du show business (Artistes United Against the War et Artists United to Win Without War), les femmes (Women's Peace Vigil, Mothers Against the War), les Noirs (Pastors for Peace, Black Voices for Peace), les syndiqués (Labor Against the War). Des organisations plus petites et plus radicales faisaient également entendre leur voix, telles que Fight Back, Direct Action, Refuse and Resist, Anti-Capitalist Convergence ou Left Turn. Les altermondialistes de Global Exchange étaient visibles, ainsi que Americans Against the War in Irak, qui dénonçait les liens de l'administration Bush avec les pétroliers. L'article de l'historien de gauche David Montgomery, intitulé «The Peace Warriors » et paru dans le Washington Post du 10 décembre 2002, donne une très bonne idée de la mosaïque formée par tous ces groupes religieux, politiques, communautaires, pacifistes, radicaux, modérés, jeunes et vieux, blancs et de couleur. Y avait-il une idéologie commune à ces différents groupes de manifestants?

Idéologies et modes d'action

D'emblée, l'absence d'idéologie commune et clairement affichée est remarquée. Les pancartes et slogans expriment de multiples opinions et tendances : le pacifisme (« The most beaufiful victory is the war we'll never launch»), le patriotisme, omniprésent ("Love my Country, Hate my Government », « Peace is Patriotic », " It's OUR Flag Too », "Peace is the American Way »), la religiosité ("God Loves People Against the War »), l'attachement aux libertés publiques («Protect Our Civil Liberties»), l'opposition directe à Bush (" Draft Dodgers Should Not Start Wars ", "Different Bush, Same Shit ", «Drop Bush Not Bombs ») et à sa politique (« Money for Jobs, not War », « No Blood for Oil»).

13 La guerre maintenant programmée en Irak rend les manifestations plus fréquentes, qui prennent pour cible la guerre, bien entendu, mais aussi les violations des droits civiques ainsi que les coupes dans les budgets sociaux. Le 15 février 2003, malgré l'interdiction de la police, 250000 personnes défilent dans les rues de New York, en synchronie avec le reste du monde. Le 8 mars, des marches de femmes sont organisées par Women for Peace devant la Maison Blanche ainsi que dans de nombreuses villes. Vêtues de rose (la journée avait reçu le nom de Pink Code), écrivaines, chanteuses, actrices, "mères et grand-mères en colère " manifestent. Les actions s'intensifient jusqu'au 20 mars 2003, date du début des actions militaires en Irak, où une véritable guerilla civique est menée par Direct Action to Stop the War à San Francisco : le centreville est bloqué et un vomit-in est organisé devant le bâtiment fédéral ; la police arrête 1 600 personnes. Ce jour-là, des manifestations se déroulent à travers tout le pays, et plus de 150 conseils municipaux de grandes et moins grandes villes votent des résolutions exprimant leur opposition à la guerre.

Dans les premiers mois de la guerre, des actions radicales sont organisées par divers groupes dont Direct Action to Stop the War, M27 Coalition (35 organisations d'extrêmegauche), ou Irak Pledge of Resistance, qui tentent de bloquer des bases militaires, casernes, centres de recrutement, entreprises fabriquant du matériel de guerre. Si ces actions restent marginales, des milliers de gens sont néanmoins arrêtés pour des actes de désobéissance civile, une expression que remettent au goût du jour la plupart des mouvements. Des manifestations de grande ampleur continuent à être organisées: le 
22 mars, 200000 personnes défilent à New York, et des actions similaires agitent San Francisco, Los Angeles ou Washington. A Salt Lake City les manifestants mettent en scène les funérailles de la démocratie; à Phoenix, un lâcher de 3000 ballons symbolisent les 3000 bombes tombées sur l'Irak. Le 25 mars, des manifestations troublent la cérémonie des Oscars. Le Monde, ce jour-là, rapporte que depuis les premières frappes en Irak les manifestations sont quotidiennes à Chicago, San Francisco, Atlanta, Los Angeles et New York. A l'époque, pourtant, un sondage réalisé par CBS montre que $70 \%$ des Américains interrogés se disent en faveur de l'action menée par Bush. Ce soutien à l'administration Bush n'est plus le même à l'automne : en octobre 2003, alors que les manifestations utilisent de plus en plus le slogan «Bring the troops home now!», 50\% des personnes interrogées désapprouvent la façon dont les Etats-Unis mènent la guerre, et 52\% estiment que la guerre en Irak n'en valait pas la peine (sondage Gallup diffusé par CNN le 27 octobre 2007). Dans le même temps, le mouvement anti-guerre s'essouffle.

Essoufflement et divisions

Dès 2003, différents journaux remarquent que le mouvement anti-guerre se divise. Les radicaux, on l'a vu, cherchent à mener des actions directes, à pratiquer la désobéissance civile. Mais les contestataires sont divisés sur les tactiques à adopter. Les modérés, tout d'abord, ont peur d'être accusés de manquer de patriotisme, et du backlash qui s'en suivrait. Même les organisations prônant la désobéissance civile et la non-violence font preuve d'une grande prudence. Win Without War, qui regroupe 36 organisations dont NOW, la NAACP, le Sierra Club, le National Council of Churches, déclare : "We want to be able to reach the mainstream of Americans, patriotic Americans, with messages and communication techniques that will resonate with them $»^{5}$. Mais les divergences portent aussi sur les objectifs: certains militants veulent marquer la différence entre " le soutien aux troupes » et " le soutien à la guerre »; certains veulent mettre l'accent sur les conséquences économiques de la guerre, par exemple la réduction des budgets sociaux, des veterans' benefits comme le souligne Youth and Student Peace Coalition. D'autres veulent se concentrer sur la reconstruction de l'Irak, tels Win Without War et MoveOn. Et d'autres, encore, veulent agir sur le Parti démocrate et soutenir des candidats anti-guerre locaux. Le mouvement, indéniablement, se divise et perd de sa dynamique. Contrairement à ce qui s'est passé pour le Vietnam, l'armée est désormais de métier; on n'appelle plus les jeunes Américains sous les drapeaux, ceux qui s'engagent sont le plus souvent des pauvres, dont beaucoup appartiennent à des minorités, et l'Amérique moyenne ne se sent ainsi pas directement menacée par la conscription. Lorsque, le 9 septembre 2004, une manifestation est organisée à New York en mémoire des victimes de la guerre en Irak, seulement 200 personnes environ se rassemblent à Columbus Circle.

Qu'est-ce que ces manifestations auront révélé sur ces mouvements, et du même coup sur les militants qui les animent?

Les spécificités du militantisme américain

17 Ces mouvements protestataires montrent tout d'abord la prolifération d'organisations et d'associations ad hoc créées pour l'occasion, coalitions, réseaux connectés par internet. Le mouvement anti-guerre est ainsi décentralisé, multiforme, sans leaders apparents. Le plus souvent, le militantisme américain, on le sait, n'a pas la forme d'un attachement à une organisation politique "formellement " identifiable. L'action de beaucoup de militants ne s'inscrit pas dans la durée (mis à part dans de notables 
exceptions). Une des caractéristiques du moment, si l'on en croit la politologue Theda Skocpol, c'est que les organisations populaires nationales existant dans le passé, et qui n'étaient pas des partis politiques, sont aujourd'hui sur le déclin ${ }^{6}$. Les militants deviennent militants dans l'action, par leur participation à des manifestations. Ils sont membres d'associations, d'advocacy groups, de mouvements plus ou moins informels (féministes, écologiques, altermondialistes...), et s'organisent en réseaux plutôt qu'en mouvements structurés. Ils font des actions ponctuelles, immédiates, ont une grande capacité à mettre sur pied des groupes ad hoc, des coalitions qui répondent à un événement, à une situation concrète, avec des objectifs précis, limités. A leur façon, ils fonctionnent comme dans des single issue movements, en défendant une cause particulière. Bon nombre de ces groupes apparaissent, disparaissent, changent de nom, mais sont capables de mobiliser des masses de gens, de se rassembler dans les " grands sursauts » dont parle François Cusset ${ }^{7}$. Leurs actions sont pragmatiques, à court terme, même si dans certains cas elles sous-tendent une vision qui englobe un avenir plus lointain. Ces militants s'investissent souvent dans la construction de " coalitions », dont le problème essentiel est qu'elles sont souvent hétéroclites, sans idéologie claire ou commune. Dans les mois qui ont suivi le 11 septembre, l'opposition à la guerre, bien que minoritaire, était présente, diffuse dans tous les secteurs de la société : même des soccer moms, même des "anciens de la CIA ", même des businessmen républicains s'y déclaraient opposés. Comment faire durer ces coalitions? Il faut un message simple, pas trop radical, des mots d'ordre qui rassemblent et taisent les divergences : « Non à la guerre », par exemple. Le mot d'ordre de la manifestation du 15 février 2003 était « The World Says No to War » : pas de mise en accusation politique, mais une recherche du consensus et une position dominante avant tout morale.

D'une certaine façon, les militants compensent l'absence de revendications politiques par une « expressivité » accrue. Ils ont souvent le souci de faire des manifestations des spectacles, soignant les mises en scènes car, comme le soulignent certains sociologues, "l'action militante devient inséparable de sa médiatisation " ${ }^{8}$. Ainsi les lâchers de ballons simulant les bombes, le transport de cercueils pour symboliser les funérailles de la démocratie, les contestataires enfermés dans des body bags de vinyl blanc pour évoquer les morts au combat, et d'autres, portant les uniformes des prisonniers d'Abou Grahib, pour dénoncer les tortures pratiquées par les Etats-Unis.

Le contexte politique de la société américaine explique cette dépolitisation. Certains traits récurrents du climat des années 2000 jouent ici un rôle prépondérant : répression sécuritaire, décrédibilisation des contestataires, parfois même criminalisation de leurs actions, servilité des médias, peur de l'opinion publique constamment manipulée, patriotisme exacerbé, soumission des élites, avec quelques exceptions. Tout cela n'est pas seulement l'effet du 11 septembre, mais également le résultat de 40 ans de guerre froide, 30 ans de gouvernements républicains (malgré les intermèdes Carter et Clinton) et le fort vent de conservatisme qui souffle sur les Etats-Unis depuis la fin des années 1960. François Cusset parle ainsi d'« espace public exsangue $»^{9}$ pour évoquer le double phénomène de dépolitisation de larges couches de la société américaine et de diabolisation de la politique quand elle n'est pas conservatrice. En témoignent le climat d'hostilité à l'engagement politique de gauche ou « radical» et le red-baiting toujours latent. A cet égard, notons une différence de taille avec l'époque du mouvement d'opposition à la guerre du Vietnam : ce mouvement était lié à l'apparition de la New Left, à un esprit d'ouverture et de tolérance à l'égard de la "vieille gauche " (refus de l'exclusion des communistes, par exemple, d'ailleurs archi-minoritaires). Aujourd'hui, 
les groupes politiques sont beaucoup moins présents. Il ne s'agit pourtant pas d'idéaliser le passé, d'oublier les tensions, les déchirures que les rivalités avaient provoquées, surtout à partir de 1969, ni les dérives sectaires du SDS et des Weathermen. D'où, ces dernières années, la volonté marquée d'empêcher tout dérapage vers la violence ou simplement vers une orientation trop marquée à gauche. C'est le consensus qui doit dominer. Il est, par exemple, significatif que le rapport des Political Research Associates de la région de Boston souligne que, sur les campus, les militants étudiants ont peur du débat ${ }^{10}$. Dans ces conditions, comment se situer sur le champ politique ? Et surtout, comment faire passer le message ? Le mouvement contestataire n'a de relais ni politique ni médiatique. D'où l'impression, comme le souligne Loïc Wacquant, que l'opposition « se voit réduite aux manifestations de rues et aux happenings symboliques, ponctuels et limités $»^{11}$, comme on le constate dans la campagne électorale de 2004.

La campagne présidentielle

Les militants s'investissent dans des manifestations lors de la Convention du Parti républicain à New York. Dans The Nation du 12 juillet 2004, la militante Liza Featherstone décrit "The Fine Art of Bush Bashing", et met l'accent sur l'aspect culturel, créatif, qui faisait l'originalité de ces manifestations, sans omettre les manifestations d'un type plus traditionnel prévues par United for Peace, ni les actions plus directes de militants anarchistes. La hantise des dérapages reste cependant très forte, les organisateurs craignant plus que tout de voir le mouvement anti-guerre discrédité et de provoquer une répression brutale.

21 Certaines organisations cherchent à élaborer de nouvelles formes de militantisme: United for Justice and Peace organise ainsi des People's Parties dans quatre quartiers de Boston lors de la Convention démocrate du 25 juillet. D'autres s'investissent dans ce qu'ils baptisent le Forum Social de Boston qui dure trois jours avec plus de 400 ateliers, séminaires, films, spectacles et expositions sur des questions telles que la justice dans le monde, les droits des immigrés ou le féminisme. Il s'agit à la fois de fournir une alternative au programme de la Convention et d'éviter les affrontements de rue, ainsi que de créer des liens entre les militants issus des classes moyennes et ceux des communautés de quartiers.

La majorité des militants anti-Bush se concentrent néanmoins sur deux types d'activités : inciter les gens à s'inscrire sur les listes électorales, et collecter des fonds pour les Démocrates. Deux organisations, MoveOn et America Coming Together, s'impliquent tout particulièrement dans ces tâches. MoveOn, du nom de son site internet (moveon.org), a été fondée en 1998 par deux entrepreneurs de software qui voulaient s'opposer à l'impeachment de Clinton et a bénéficié des largesses du financier George Soros. Cette organisation qui utilise essentiellement l'internet se révèle rapidement très efficace dans la collecte de fonds. America Coming Together, dirigée par Steve Rosenthal, un ancien responsable de l'AFL-CIO, déploie un vaste réseau de délégués qui font du porte-à-porte, surtout dans les quartiers ouvriers, pour inciter les électeurs proches du parti démocrate à voter. Harold Meyerson, un syndicaliste, affirme ainsi que America Coming Together a collecté 135 millions de dollars et a convaincu des millions de gens, dans des quartiers pauvres, noirs, délaissés, de voter Kerry. Meyerson voit cet exploit comme «the restoration of community-based politics ${ }^{12}$. Mais est-ce bien si évident ? Les organisateurs de ACT sont extérieurs aux quartiers, issus des classes moyennes, et sont rémunérés. 

mais sans analyser le contenu du programme du Parti démocrate et sans le contester d'aucune façon. John Kerry a finalement obtenu 6,2 millions de voix de plus qu'Al Gore, mais avec quel programme ? Le seul candidat à se poser cette question est Ralph Nader. Le type d'actions organisées par America Coming Together et MoveOn nous renseigne finalement sur les formes du militantisme politique dans un système où le bipartisme est dominant, où les deux partis convergent sur de nombreux points, où la personnalisation du scrutin présidentiel est aussi forte, l'argent aussi déterminant, le taux d'abstention aussi élevé et le culte de la technologie aussi prégnant. Dans le même article, Harold Meyerson va jusqu'à avancer : « The 527 era has turned out to be one of renewed grass-roots activism and small-donor participation $»^{13}$. La collecte d'argent et l'ordinateur seraient-ils devenus les mamelles du militantisme? «From the Dean campaign to MoveOn, from America Votes to America Coming Together", écrit encore Meyerson, «a new generation of activists is bent on rebuilding, if not the Democratic Party, at least the Democratic majority, through the new-old magic of grassroots activism $»^{14}$. Est-ce là l'horizon du militantisme américain ? Doit-on penser que l'avenir du militantisme passe par internet et que, grâce à des organisations telles que MoveOn et $A C T$, on va voir à l'œuvre «the new-old magic of grassroots activism » ? Le recours à la technologie, qui permet, certes, une diffusion de l'information et une mobilisation rapides, masque l'essentiel, à savoir la dimension politique du problème de fond. Faut-il vraiment célébrer «la démocratie internet»? Comme l'écrit ironiquement George Packer dans le New York Times Magazine du 9 mars 2003 : «Internet democracy solves the problem of how to focus political activity in a vast country of extremely busy and distracted citizens because what keeps so many Americans busy and distracted these days is the internet $»^{15}$.

24 La conception de la politique et du militantisme qui se profile derrière ce discours et derrière l'action de MoveOn peut ainsi apparaître comme dangereuse. Les positions politiques des citoyens seraient, en quelque sorte, préconstituées, et il ne s'agit plus que de mettre ces citoyens en réseaux et de cliquer sur la bonne touche. George Packer, dans le même article, cite l'un des fondateurs de MoveOn et explique à son propos : « [...] he and other organizers are less political propagandists than 'facilitators' who help people to do what they want to do ». Le militantisme se résume-t-il à cela ? Militer ne signifie-t-il pas se forger une vision du monde et de la société dans l'expérience de la lutte, un apprentissage de la solidarité dans l'action collective sur le terrain, une confrontation permanente avec la réalité de la lutte des classes, la lutte contre l'injustice, la lutte anti-impérialiste? Le militantisme a un rôle essentiel dans l'éducation, la formation politique. Assistons-nous à l'apparition d'un OVNI militant? La nouvelle promesse du militantisme de la base, "grassroots activism », serait-elle la démocratie électronique? Ou bien les militants sauront-ils résister pour reconstruire une alternative au Parti démocrate actuel et pour retrouver les chemins de l'expérience militante et de la solidarité ?

Le mouvement anti-guerre d'après le 11 septembre reflète bien quelques caractéristiques du militantisme américain. Ce mouvement est une nébuleuse à l'intérieur de laquelle existe une grande diversité d'opinions. C'est plutôt le rassemblement de " communautés de conscience », dont a parlé Edward Said ${ }^{16}$, qu'un rassemblement de militants politiques. L'ampleur de certaines manifestations, la rapidité avec laquelle elles apparaissent puis déclinent avant de disparaître, sont 
frappantes. Ce caractère éphémère révèlerait-il que les convictions des militants/ participants sont également éphémères ? Pas nécessairement. Il y a de la continuité dans les convictions de certains si l'on constate que, par exemple en février 2005, lors d'un rassemblement de United for Peace and Justice qui regroupait alors 1000 organisations, un tiers des militants avaient participé au mouvement des années soixante (droits civiques, opposition à la guerre du Vietnam). On pourrait citer des participants célèbres, Daniel Ellsberg, Susan Sontag, Howard Zinn et d'autres. Ne peuton pas plutôt expliquer cette apparente discontinuité par le style actuel du militantisme et surtout par l'absence de relais politiques, qui entraînent découragement ou frustration devant la difficulté de changer le cours des choses?

On doit, en tout cas, souligner la marginalité durable des groupes militants, qui explique en partie leur comportement. Doit-on aller aussi loin que Loïc Wacquant, lorsqu'il dit de l'opposition qu'elle « tourne en quelque sorte à vide : elle est dépourvue de relais efficients dans les champs journalistique et politique... Elle n'a pas les moyens de traduire cette mobilisation en pression sur l'appareil d'Etat, car celui-ci est parfaitement isolé de la société par une machine électorale contrôlée par les intérêts d'argent $\aleph^{17}$ ? Les minorités agissantes n'auraient-elles plus leur place dans le contexte américain?

\section{NOTES}

1. Voir Jacques Ion, Spyros Franguiadakis et Pascal Viot, Militer aujourd'hui (Paris : Collection CEVIPOF/ Autrement, 2005), ainsi que Danielle Tartakowsky, La Manif en éclats (Paris : La Dispute, 2004).

2. David Usborne, «Campuses divided as anti-war lobby grows », The Independent (22 Septembre 2003), 11.

3. Amy Goodman et David Goodman, The Exception to the Rulers. Exposing Oily Politicians, War Profiteers and the Media that Love Them (New York : Hyperion, 2004), 20.

4. Goodman et Goodman, The Exception to the Rulers, 147-148.

5. Le Monde, Supplément New York Times (23-24 mars 2003), 3.

6. Theda Skocpol, « Les milieux populaires n'ont plus de voix », Alternatives économiques (septembre 2004), 39.

7. François Cusset, « Les états désunis de la gauche américaine », Mouvements (novembre-décembre 2003), 50-51.

8. Ion, Franguiadakis, et Viot, Militer aujourd'hui, 8.

9. Cusset, « Les états désunis de la gauche américaine », 49.

10. Pam Chamberlain, «Debate is unpopular on campus », in Pam Chamberlain, Deliberate Differences: Progressive and Conservative Campus Activism in the United States (Somerville, Mass : Political Research Associates, 2004), 28.

11. Loïc Wacquant, « L'ordre nu de l'hyperpuissance », L'Humanité (9 avril 2003), 22-23.

12. Harold Meyerson, «Whither the Ward Heelers?", The American Prospect (janvier 2005), 11.

13. Meyerson, « Whither the Ward Heelers?». 
14. Meyerson, « Whither the Ward Heelers? », 13.

15. George Packer, « Smart-Smobbing the War », The New York Times Magazine (9 mars 2003), 48.

16. Edward Said, « Une autre façon de voir les Etats-Unis », Le Monde Diplomatique (mars 2003), 21.

17. Loïc Wacquant, « L'ordre nu de l'hyperpuissance », 22-23. 